\title{
COMMENTARY
}

\section{Contrast-induced nephropathy: attributable incidence and potential harm}

\author{
Lilian Pires de Freitas do Carmo and Etienne Macedo* \\ See related research by Cely et al., http://ccforum.com/content/16/2/R67
}

\begin{abstract}
Contrast-induced nephropathy is a common form of hospital-acquired acute kidney injury. Incidence is low in patients with normal renal function but increases in high-risk patients. Patients with contrast-induced nephropathy have higher in-hospital complication rates and mortality. Critically ill patients have been assumed to be a high-risk group for contrast-induced nephropathy. In the previous issue of Critical Care, Cely and colleagues showed an unexpectedly low incidence of contrast-induced nephropathy in critically ill patients receiving radiographic contrast material for computerized tomography. We should note that it is difficult to establish the true frequency and impact of the contrast nephrotoxicity because of many other causes for acute kidney injury in this population. Moreover, the impact on long-term kidney function and the possible effect of this insult on the recovery of renal function when associated with other causes of acute kidney injury are unknown.
\end{abstract}

Contrast-induced nephropathy (CIN) is a known complication of intravenous, iodinated contrast and is a common cause of acute kidney injury (AKI) in the inpatient setting. The reported incidence of CIN varies among studies, due to differences in definition, type and dose of contrast medium, study population, imaging procedure and the frequency of other potential causes of AKI [1]. The widely used definition for CIN is as an acute rise of $0.5 \mathrm{mg} / \mathrm{dl}$ in serum creatinine or a relative increase of more than $25 \%$ from the baseline value. CIN is usually transient, with serum creatinine beginning to rise within 24 to 48 hours after exposure, reaching a peak within 3 to 5 days after the administration of the contrast medium,

*Correspondence: etimacedo@usp.br

Department of Internal Medicine, Division of Nephrology, University of São

Paulo School of Medicine, Av Dr Eneas de Carvalho Aguiar, 255 sala 11 F-7 andar, Sao Paulo, SP 05403-000, Brazil and returning to baseline within 7 to 10 days after administration [2]. The pathogenesis of CIN is not completely defined, and suggests a combination of toxic injury to the renal tubules and ischemic injury, partly mediated by reactive oxygen species [3].

The CIN incidence can range from $2 \%$ to more than $40 \%$ in high-risk patients [4-9]. Although the reported need for acute hemodialysis is low [1,10], patients developing CIN have longer hospital stays, more complicated clinical courses and higher mortality rates $[1,4]$. The relationship of CIN to long-term adverse events is controversial. Only a small number of studies report kidney function beyond a few days after exposure to the contrast medium. In one study, the rate of long-term adverse events was higher in individuals with CIN after adjustment for baseline comorbidities and risk factors [11]. Although these data signal a worse long term in patients with contrast nephropathy, there is still insufficient evidence to reach a definitive conclusion.

Pre-existing chronic kidney disease is the greatest risk factor in the development of CIN. Other factors include diabetes, age over 75 years, heart failure, cirrhosis, hypertension, anemia, intra-arterial injection, hypotension, intra-aortic balloon pump and volume of contrast [12]. Most of these risk factors are often found in patients who have been admitted to the ICU. Additionally, computerized tomography with intravenous administration of iodinated radiographic contrast media is a frequent and necessary procedure for the management of critically ill patients in a variety of situations. However, the risk for CNI from radio-contrast exposure has not been well evaluated in this population.

In the previous issue of Critical Care, Cely and colleagues performed a single-center prospective matched cohort study of ICU patients requiring CT scanning with or without radio contrast to determine the incidence of CIN [13]. They matched patients based on pre-scan measured creatinine clearance, diabetes and mechanical ventilation. The primary end point was a decrease in measured creatinine clearance by $33 \%$ within 3 days following scanning. The decline in renal function was equally frequent in groups scanned with and without 
radiographic contrast material (RCM). Without a control group who were not exposed to contrast, the decreased glomerular filtration rate following the RCM was attributed to CIN. However, a similar rate of decline in renal function experienced by patients scanned without RCM leads to the conclusion that the loss of glomerular filtration rate in this scenario cannot be attributed solely to the RCM exposure.

A frequent decline in renal function and substantial daily variation in serum creatinine is known to occur in critically ill patients, whether or not they have been exposed to contrast-media injections [14]. Depending on the criteria in use, these fluctuations in serum creatinine can determine different incidences of $\mathrm{CIN}$-associated AKI. The impact of the fluctuations of renal function in critically ill patients on the diagnosis of CIN has also been discussed by Bruce and colleagues [15]. Considering these findings and the results of the present study by Quartin and colleagues, it becomes clear that AKI after the administration of RCM may be reflective of other processes occurring simultaneously in the critically ill patient. In this context, the diagnosis of CIN is challenging. Instead of ruling out $\mathrm{CIN}$, other possible causes of AKI should be considered. Therefore, patients suspected of CIN must also be evaluated for other possible causes of AKI [16].

Before we accept a low incidence of CIN in critically ill patients, other important factors should be mentioned. The majority of patients in the study by Quartin and colleagues received CIN prophylaxis. The average fluid administration exceeded 31 on the day of scanning among both contrast patients and noncontrast patients. CIN is a preventable cause of AKI and, even if the incidence in ICU patients may be low, prophylaxis should always be carried out. Furthermore, CIN has unfavorable outcomes and the long-term outcomes in patients with CIN are uncertain. Whether CIN can increase the severity of AKI and the time to recover renal function is still unknown.

Identification of earlier and etiologic-specific biomarkers for CIN will probably allow determination of the real incidence of CIN and the development of targeted therapies. Currently, the use of delta creatinine in a 12-hour period after contrast exposure could be an alternative with good sensitivity and specificity for early detection [17]. While waiting for a more sensitive and specific biomarker for CIN, clinicians should weigh the benefit of scanning with contrast media against the shortterm and long-term risks of CIN-associated AKI.

\section{Abbreviations \\ AKI, acute kidney injury; CIN, contrast-induced nephropathy; RCM, radiographic contrast material.}

Competing interests

The authors declare that they have no competing interests.
Published: 23 May 2012

\section{References}

1. McCullough PA, Wolyn R, Rocher LL, Levin RN, O'Neill WW: Acute renal failure after coronary intervention: incidence, risk factors, and relationship to mortality. Am J Med 1997, 103:368-375.

2. Rich MW, Crecelius CA: Incidence, risk factors, and clinical course of acute renal insufficiency after cardiac catheterization in patients 70 years of age or older. A prospective study. Arch Intern Med 1990, 150:1237-1242.

3. Katholi RE, Woods WT, Taylor GJ, Deitrick CL, Womack KA, Katholi CR, McCann WP: Oxygen free radicals and contrast nephropathy. Am J Kidney Dis 1998, 32:64-71.

4. Marenzi G, Lauri G, Assanelli E, Campodonico J, De Metrio M, Marana I, Grazi $M$, Veglia F, Bartorelli AL: Contrast-induced nephropathy in patients undergoing primary angioplasty for acute myocardial infarction. J Am Coll Cardio/ 2004, 44:1780-1785.

5. Briguori C, Airoldi F, D'Andrea D, Bonizzoni E, Morici N, Focaccio A, Michev I, Montorfano M, Carlino M, Cosgrave J, Ricciardelli B, Colombo A: Renal Insufficiency Following Contrast Media Administration Trial (REMEDIAL): a randomized comparison of 3 preventive strategies. Circulation 2007, 115:1211-1217.

6. Marenzi G, Assanelli E, Marana I, Lauri G, Campodonico J, Grazi M, De Metrio M, Galli S, Fabbiocchi F, Montorsi P, Veglia F, Bartorelli AL: $N$-acetylcysteine and contrast-induced nephropathy in primary angioplasty. N Eng/ J Med 2006, 354:2773-2782

7. Tepel M, van der Giet M, Schwarzfeld C, Laufer U, Liermann D, Zidek W: Prevention of radiographic-contrast-agent-induced reductions in renal function by acetylcysteine. N Eng/ J Med 2000, 343:180-184.

8. Mitchell $A M$, Jones AE, Tumlin JA, Kline JA: Incidence of contrast-induced nephropathy after contrast-enhanced computed tomography in the outpatient setting. Clin J Am Soc Nephrol 2010, 5:4-9.

9. Huber W, Eckel F, Hennig M, Rosenbrock H, Wacker A, Saur D, Sennefelder A, Hennico R, Schenk C, Meining A, Schmelz R, Fritsch R, Weiss W, Hamar P, Heemann U, Schmid RM: Prophylaxis of contrast material-induced nephropathy in patients in intensive care: acetylcysteine, theophylline, or both? A randomized study. Radiology 2006, 239:793-804.

10. Freeman RV, O'Donnell M, Share D, Meengs WL, Kline-Rogers E, Clark VL, DeFranco AC, Eagle KA, McGinnity JG, Patel K, Maxwell-Eward A, Bondie D, Moscucci M: Nephropathy requiring dialysis after percutaneous coronary intervention and the critical role of an adjusted contrast dose. Am J Cardiol 2002, 90:1068-1073.

11. Solomon RJ, Mehran R, Natarajan MK, Doucet S, Katholi RE, Staniloae CS, Sharma SK, Labinaz M, Gelormini JL, Barrett BJ: Contrast-induced nephropathy and long-term adverse events: cause and effect? Clin J Am Soc Nephrol 2009, 4:1162-1169.

12. Mehran R, Aymong ED, Nikolsky E, Lasic Z, lakovou I, Fahy M, Mintz GS, Lansky AJ, Moses JW, Stone GW, Leon MB, Dangas G: A simple risk score for prediction of contrast-induced nephropathy after percutaneous coronary intervention: development and initial validation. J Am Coll Cardio/ 2004, 44:1393-1399.

13. Cely CM, Schein RMH, Quartin AA: Risk of contrast induced nephropathy in the critically ill: a prospective, case matched study. Crit Care 2012, 16:R67.

14. Newhouse JH, Kho D, Rao QA, Starren J: Frequency of serum creatinine changes in the absence of iodinated contrast material: implications for studies of contrast nephrotoxicity. AJR Am J Roentgeno/ 2008, 191:376-382.

15. Bruce RJ, Djamali A, Shinki K, Michel SJ, Fine JP, Pozniak MA: Background fluctuation of kidney function versus contrast-induced nephrotoxicity. AJR Am J Roentgenol 2009, 192:711-718.

16. KDIGO Acute Kidney Injury Work Group: KDIGO clinical practice guideline for acute kidney injury. Kidney Int Supp/ 2012, 2:1-138.

17. Ribichini F, Gambaro G, Graziani MS, Pighi M, Pesarini G, Pasoli P, Anselmi M, Ferrero V, Yabarek T, Sorio A, Rizzotti P, Lupo A, Vassanelli C: Comparison of serum creatinine and cystatin $C$ for early diagnosis of contrast-induced nephropathy after coronary angiography and interventions. Clin Chem 2012, 58:458-464.

doi:10.1186/cc11327

Cite this article as: Pires de Freitas do Carmo L, Macedo E.: Contrast-induced nephropathy: attributable incidence and potential harm. Critical Care 2012, 16:127. 